\title{
artigo
}

\section{Método para implantação de um observatório para sífilis gestacional e congênita: relato de experiência}

\author{
Method for setting up an observatory for gestational and congenital syphilis: experience report \\ Método para establecer un observatorio para gerentes y congénitos: un informe de experiencia
}

\begin{abstract}
RESUMO
Objetivo: descrever o método de implantação de um observatório para sífilis gestacional e congênita. Método: Estudo descritivo, realizado no município de Londrina, Paraná/Brasil, no período de 2013 a 2016. A implantação do observatório se deu em seis etapas: 1) formação de grupo de trabalho; 2) diagnóstico situacional 3) educação permanente; 4) estruturação da linha de cuidado e comunicação; 5) adequação dos procedimentos técnicos laboratoriais; 6) ordenação do processo de trabalho. Resultados: As ações implantadas impactaram no aumento da detecção de casos de sífilis em gestantes na atenção básica, que passaram de 9,4 a cada mil nascidos vivos em 2013 para 16,7 em 2015. A mortalidade específica por sífilis congênita alcançou 41,7 casos por 100 mil nascidos vivos em 2013 e reduziu para zero nos anos subsequentes. Conclusão: A criação do observatório permitiu a ampliação do diagnóstico da sífilis na gestação, melhoria da assistência materno-infantil e redução da transmissão vertical da sífilis.
\end{abstract}

DESCRITORES: Observatório de Saúde; Sífilis Congênita; Cuidado Pré-Natal; Atenção Primária a Saúde.

\section{ABSTRACT}

Objective: to describe the method of implanting an "Observatory for gestational and congenital syphilis". Method: Descriptive study, carried out in the city of Londrina, Paraná, Brazil, from 2013 to 2016. To implement the observatory, it was necessary to comply with the following steps: 1) formation of the syphilis working group; 2) situational diagnosis; 3) permanent education workshops; 4) structuring the line of care and communication; 5) adequacy of technical laboratory procedures for performing the Venereal Disease Research Laboratory; 6) ordering the work process in obstetric care. Results: The actions implemented impacted the increase in the detection of syphilis cases in pregnant women in primary care, which went from 9.4 per thousand live births in 2013 to 16.7 in 2015. The specific mortality from congenital syphilis reached 41.7 cases per 100,000 live births in 2013 and reduced to zero in subsequent years. Conclusion: The creation of the observatory allowed the expansion of the diagnosis of syphilis during pregnancy, improved maternal and child care and reduced vertical transmission of syphilis.

DESCRIPTORS: Health Observatory; Syphilis, Congenital; Prenatal Care; Primary Health Care.

\section{RESUMEN}

Objetivo: describir el método de implantación de un observatorio de sífilis gestacional y congénita. Método: Estudio descriptivo, realizado en la ciudad de Londrina, Paraná / Brasil, de 2013 a 2016. La implementación del observatorio se realizó en seis etapas: 1) formación de un grupo de trabajo; 2) diagnóstico situacional 3) desarrollo de talleres de educación permanente; 4) estructuración de la línea de atención y comunicación; 5) adecuación de los procedimientos técnicos de laboratorio; 6) ordenar el proceso de trabajo. Resultados: Las acciones implementadas impactaron el aumento en la detección de casos de sífilis en mujeres embarazadas en atención primaria, que pasó de 9.4 por mil nacidos vivos en 2013 a 16.7 en 2015. La mortalidad específica por sífilis congénita alcanzó 41.7 casos por cada 100,000 nacimientos vivos en 2013 y reducido a cero en años posteriores. Conclusión: La creación del observatorio permitió la expansión del diagnóstico de sífilis durante el embarazo, mejoró el cuidado materno-infantil y redujo la transmisión vertical de sífilis.

DESCRIPTORES: Observatorio de Salud; Sífilis Congénita; Cuidado prenatal; Atención Primaria de Salud.

RECEBIDO EM: 14/12/2020 APROVADO EM: 11/01/2021

\section{Flaviane Mello Lazarini}

Professor Adjunta, Departamentos de Enfermagem, Universidade da Região de Joinville, UNIVILLE, ORCID: 0000-0003-1325-2744 


\section{Guilherme Cassins Valdez}

Graduandos em Medicina, Universidade da Região de Joinville, UNIVILLE. ORCID: 0000-0003-2816-2805

\section{Luan Gabriel Bueno Valgas}

Graduandos em Medicina, Universidade da Região de Joinville, UNIVILLE. ORCID: 0000-0002-9710-2382

\section{Marcos Vinícius Maruyama Ribeiro}

Graduandos em Medicina, Universidade da Região de Joinville, UNIVILLE. ORCID: 0000-0003-3627-4110

\section{Pietro Domit}

Graduandos em Medicina, Universidade da Região de Joinville, UNIVILLE. ORCID: 0000-0003-1972-7392

\section{Luciano Henrique Pinto}

Professor Adjunto, Departamentos de Medicina, Enfermagem e Farmácia, Universidade da Região de Joinville, UNIVILLE, Coordenador do Projeto Integrado ECOSAM.

ORCID: 0000-0003-0250-7502

\section{INTRODUÇÃO}

$\square$ m 2010, a Organização Mundial de Saúde (OMS) estimou que dos mais de dois milhões de casos anuais de sífilis gestacional em todo o globo, aproximadamente $25 \%$, ocorreram na América Latina e no Caribe, onde a prevalência de sífilis gestacional chegou a 3,9\%, acima da média mundial de $1,8 \%{ }^{1}$.

De acordo com a Organização Pan-Americana de Saúde (OPAS), até 2015, a meta era reduzir a sífilis congênita para menos de 0,5 casos/mil nascidos vivos ${ }^{2}$. Esta meta está longe de ser alcançada no Brasil, uma vez que dados nacionais indicaram incidência de 4,7 casos de sífilis congênita por mil nascidos vivos. No mesmo ano, a cidade de Londrina localizada no estado do Paraná, superou os índices nacionais $(7,1 / 1000)^{1}$.

A epidemia global da sífilis gerou a necessidade de ampliar o monitoramento das taxas de transmissão vertical da doença em âmbito local, e realizar intervenções junto à gestão na Rede de Atenção à Saúde (RAS) materno-infantil. Neste contexto, o observatório se constitui em uma ferramenta de apoio à gestão de políticas e sistemas de saúde, reconhecida em âmbito internacional ${ }^{3}$.

Considerando, que o quinto Objetivo de Desenvolvimento do Milênio (ODM), que buscou melhorar a saúde materna no
Brasil e no mundo até 2015 não foi atingido ${ }^{4}$ e que a proposta da OPAS para eliminação da transmissão vertical necessita de estratégias factíveis, com políticas que incentivem a identificação e a correção das falhas geradoras da sífilis de acordo com às distintas realidades regionais brasileiras ${ }^{5}$, surge a necessidade de propor e implantar um observatório para sífilis congênita e gestacional em um município brasileiro com alta incidência da doença com vistas a aperfeiçoar a assistência pré-natal local e consequentemente reduzir a transmissão vertical da sífilis.

O objetivo deste estudo é descrever o método de implantação de um observatório para sífilis gestacional e congênita e relatar a experiência obtida nesta experiência.

\section{MÉTODO}

Trata-se de estudo descritivo de análise de processo de implantação de um observatório para sífilis congênita e neonatal no município de Londrina PR. Este município, situa-se ao Norte do Paraná, Brasil, sendo considerada a segunda cidade mais populosa do Estado. Com 80 anos e crescimento constante, o município firmou-se como principal referência regional. Em 2010, a população residente em Londrina alcançou 506.701 habitantes ${ }^{6}$.

O observatório foi implantado no perí- odo de 2013 a 2015, como ferramenta de vigilância epidemiológica e foi posteriormente incorporada à gestão municipal para controle e monitoramento da sífilis.

Sua implantação foi conduzida por pesquisadores da Universidade Estadual de Londrina e por 102 profissionais de saúde atuantes na atenção primária, média e alta complexidade do município. A análise e estudo dos resultados foram feitos por professores e alunos da Universidade da Região de Joinville-SC, envolvidos em projeto sífilis.

A implantação do observatório se deu em seis etapas: 1) Formação grupo de trabalho sífilis; 2) Diagnóstico situacional por meio do levantamento dos problemas existentes na rede de atenção à saúde; 3 ) Desenvolvimento de oficinas de educação permanente para a Atenção Básica; 4) Estruturação da linha de cuidado e da comunicação entre os diferentes pontos de atenção; 5) Adequação dos procedimentos técnicos laboratoriais para a realização do Venereal Disease Research Laboratory (VDRL) ; 6) Ordenação do processo de trabalho na assistência obstétrica.

Critérios de inclusão adotados foram: ser profissional de saúde vinculado aos serviços de atenção básica e materno infantil da secretaria de saúde de Londrina, graduado ou bacharel. Critério de exclusão: estudantes de graduação que participaram foram excluídos. A avaliação do questio- 
nário foi realizada levando-se em consideração as respostas corretas e incorretas de acordo com o referencial que sustentou o treinamento.

O estudo foi precedido da aprovação do Comitê de Ética em Pesquisa (CEP) da Unifesp (parecer no 520.189 de 12/02/2014) e da assinatura do Termo de Consentimento Livre e Esclarecido (TCLE) pelos profissionais incluídos na pesquisa.

\section{RESULTADOS}

A implantação do Observatório para Sífilis Gestacional e Congênita no município de Londrina, Paraná, foi estruturado em seis etapas, apresentadas na Figura 1.

A seguir estão descritas detalhadamente cada uma das etapas do método para a implantação dessa ferramenta de gestão.

\section{Formação grupo de trabalho (GT) sífilis}

Com a finalidade de apoiar as intervenções realizadas pelo observatório, em outubro de 2013 ocorreu a criação do Grupo de Trabalho Sífilis (GT sífilis), composto por dois médicos e seis enfermeiros, atuantes no Comitê Municipal de Prevenção da Mortalidade Materna e Infantil (CMPMMI), na Diretoria de Atenção Primária à Saúde (DAPS), nas Diretorias de Vigilância em
Saúde (DVS) e de Serviços Especiais (Centro de Testagem e Aconselhamento - CTA e a Maternidade) e na Universidade Estadual de Londrina. Com periodicidade de uma reunião por mês, o grupo levantou dados epidemiológicos e elencou os problemas na Rede de Atenção à Saúde (RAS) local.

\section{Diagnóstico situacional por meio do levantamento dos problemas exis- tentes na rede de atenção à saúde}

O levantamento de dados aconteceu por meio dos casos de óbitos fetais e infantis que eram investigados CMPMMI e também por dados epidemiológicos dos sistemas de informação Sistema de Informação sobre Mortalidade (SIM) e Sistema de Informação de Agravos de Notificação (SINAN). Desta forma foram enumerados os seguintes impasses:

a. O número de óbitos fetais e infantis por sífilis estava aumentando anualmente;

b. Os fatores relacionados à boa parte dos casos de sífilis congênita eram o início tardio do pré-natal, vulnerabilidade social e dificuldade em diagnosticar e tratar as parceiros sexuais das gestantes;

c. Trabalho fragmentado na assistência, casos de sífilis gestacional

Figura 1. Etapas de estruturação do Observatório para Sífilis Gestacional e Congênita, Londrina, Paraná, Brasil

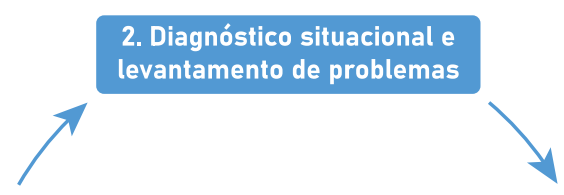

1. Formação do grupo de trabalho da siffilis

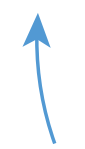

5. Ordenação do processo de trabalho na assistência obstétrica
3. Oficinas de Educação Permanente para a Atenção Básica

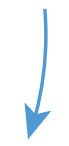

4. Estruturação da linha de cuidado e da comunicação entre a Rede de Atenção a Saúde no território das Unidades Básicas de Saúde que não estavam sendo acompanhados;

d. Falta de confiabilidade dos testes de VDRL, problema que foi identificado com a repetição de testes VDRL positivos no momento do parto em gestantes que tinham três testes negativos no pré-natal;

e. Desarticulação da Rede de Atenção para pré-natal, parto e puerpério;

f. Ausência de retroalimentação de informações entre os níveis de assistência (referência e contrarreferência);

g. Atrasos de notificação e preenchimento inadequado da ficha;

h. Ausência de fluxo local para seguimento do recém-nascido exposto à sífilis.

\section{Desenvolvimento de oficinas de edu-} cação permanente para a atenção básica (AB)

A partir do diagnóstico situacional duas frentes de trabalho foram traçadas. A primeira direcionada à educação permanente dos profissionais da assistência materno-infantil local e a segunda voltada à organização da RAS local, instituindo referência, contrarreferência e monitoramento dos indicadores epidemiológicos.

O enfoque principal do observatório foi a $\mathrm{AB}$, por isso, houve capacitação dos 102 profissionais que se tornaram multiplicadores. Esses treinamentos ocorreram em três etapas. Os facilitadores formados replicaram as oficinas de educação permanente em 50 das 54 UBS de Londrina.

As oficinas trabalharam principalmente com a notificação dos indivíduos infectados com sífilis, com ênfase no preenchimento adequado das fichas de sífilis gestacional e sífilis congênita, adequação do processo de trabalho para envio rápido das notificações para o setor de epidemiologia, diagnóstico e manejo da sífilis de acordo com os protocolos ministeriais vigentes e responsabilização pelos casos ocorridos nos territórios de abrangência das Unidades de Saúde.

Ao final de cada intervenção educacional, com apoio de fluxogramas descritores 
do processo de trabalho, as próprias equipes identificavam os erros cometidos no pré-natal por meio da discussão de casos clínicos reais de óbitos fetais e abortos evitáveis causados por sífilis.

Paralelamente ao treinamento, a partir de janeiro de 2014, iniciou-se a notificação da sífilis adquirida pelas UBS locais, uma vez que não ocorria formalmente em ficha de notificação própria. No mesmo período, tornou-se vigente o "Aprazamento", documento para acompanhamento das gestantes com sífilis na $\mathrm{AB}$, produzido em duas vias, uma anexada ao prontuário e outra ao cartão da gestante.

$\mathrm{O}$ instrumento de referência contém as datas do esquema de tratamento preconizado de acordo com o estadiamento da sífilis, os resultados do acompanhamento mensal da titulação VDRL e a informação de localização e tratamento do parceiro sexual. Em janeiro de 2016 a 17ª Regional de Saúde do Paraná padronizou esse instrumento para todos os seus 21 municípios.

\section{Estruturação da linha de cuidado e da comunicação entre os diferentes pontos da Rede de Atenção à Saúde.}

Essa frente de trabalho buscou estruturar a linha de cuidado local para atendimento integral na Rede de Atenção preestabelecida. Considerando a necessidade de impedir a cadeia de transmissão da sífilis por meio da prevenção e do tratamento adequado, estratégias populacionais foram disparadas durante os três anos de trabalho do observatório (2014 a 2016).

$O$ Centro de Referência para testagem e aconselhamento em parceria com a $\mathrm{AB}$ realizaram campanhas de testagem para sífilis e outras infecções sexualmente transmissíveis (IST), junto com as campanhas de coleta de citologia oncótica para prevenção de câncer de colo de útero e mama nas UBS. Também foi elaborado e distribuído um panfleto instrutivo sobre síflis para a população, contendo medidas de prevenção, como o uso do preservativo, a importância do diagnóstico e do tratamento dos parceiros e consequências da transmissão vertical para o feto, disponibilizado em todas as Unidades de Saúde.
Para cobrir a assistência obstétrica, tanto a Maternidade Municipal, referência para partos de gestações de risco habitual e intermediário e que atende em média $75 \%$ dos partos de Londrina realizados pelo Sistema Único de Saúde (SUS), quanto os três hospitais com maternidades particulares e conveniadas receberam treinamento para médicos e enfermeiros abordando a importância dos testes rápidos de vírus da imunodeficiência humana (VIH) e sorologia para sífilis no pré-parto.

A cobertura da Maternidade Municipal para esses testes é de $100 \%$ dos partos, porém para as outras instituições a realização do VDRL ou do teste rápido não era inserida na rotina. A Maternidade Municipal implantou fluxos para organizar o processo de trabalho: "Triagem da Parturiente", "Recém-Nascidos (RN) de Mães com Síflis Adequadamente Tratadas", "Recém-Nascidos de Mães com Sífilis não Tratadas ou Inadequadamente Tratadas". O material foi disponibilizado para as outras instituições para que pudessem adaptar suas práticas às normas ministeriais preconizadas.

\section{Adequação dos procedimentos técni- cos laboratoriais para a realização do Venereal Disease Research Labora- tory (VDRL)}

Com apoio da vigilância sanitária municipal os representantes de laboratórios de análises clínicas públicos e privados receberam treinamento sobre a importância de seguir a normatização nacional, de acordo com a Portaria Ministerial no 3.242, de 30 de dezembro de $2011^{5}$, uma vez que se identificou que algumas instituições não estavam utilizando o número de kits adequados (dois) para determinação de anticorpos e confirmação da titulação VDRL.

Após a adequação técnica laboratorial também foi instituída mudança na comunicação e processo de trabalho laboratório/ UBS, oficializada por comunicação interna que recomendou em casos de exames VDRL positivos: o laboratório envia e-mail para a UBS informando o nome da gestante, número do cartão SUS e o resultado. O laboratório passou a guardar o frasco de sorologia, esperando a resposta da Unidade.
A UBS responde o e-mail com as seguintes informações: se é para encaminhar a amostra para a realização de FTA-Abs (Fluorescent Treponemal Antibody - Absorption) ou se trata-se de controle de titulação.

\section{Ordenação do processo de trabalho na assistência obstétrica}

Simultaneamente os hospitais foram orientados a realizar o VDRL em mulheres que tivessem abortos espontâneos e nos casos de óbitos fetais. Para esse controle, o Hospital Universitário Regional do Norte do Paraná (HU), referência para emergências obstétricas, desenvolveu um carimbo de VDRL utilizado nos prontuários desses casos. Institui-se que no pronto socorro obstétrico as mulheres receberiam alta apenas quando seus prontuários tivessem recebido o carimbo.

Foi estabelecida a contrarreferência na Maternidade Municipal por meio de e-mail institucional padronizado para as UBS. Todos os nascidos vivos de mulheres que tiveram sífilis na gestação passaram a ser encaminhados para o ambulatório de referência em moléstias infecciosas pediátricas.

Além disso, para cada recém-nascido exposto à sífilis, mesmo quando a mãe foi adequadamente tratada na gestação, a $\mathrm{CCIH}$ da Maternidade de referência envia e-mail para a UBS da área de abrangência, informado dados da mãe e da criança e data da consulta agendada no ambulatório moléstias infecciosas pediátricas.

Cabe à equipe de cada UBS, monitorar o comparecimento do binômio à consulta com especialista, bem como, o enfermeiro responsável pela área da puérpera, acompanhar de perto o crescimento e desenvolvimento da criança por meio da puericultura, além da realização de titulação mensal de VDRL até o bebê receba alta.

\section{DISCUSSÃO}

A implantação do observatório e as açốes implantadas impactaram no aumento da detecção de casos de sífilis em gestantes na atenção básica, que passaram de 9,4 a cada mil nascidos vivos em 2013 para 16,7 em 2015. A mortalidade específica por síf- 


\section{artigo}

Lazarini, F.M.; Valdez, G.C.; Valgas, L.G.B.; Ribeiro, M.V.M.; Domit, P.; Pinto, L.H.;

Método para implantação de um observatório para sífilis gestacional e congênita: relato de experiência

lis congênita alcançou 41,7 casos por 100 mil nascidos vivos em 2013 e reduziu para zero nos anos subsequentes.

Além disto podemos destacar que os principais benefícios do Observatório para comunidade foram o fornecimento de subsídios para monitorar a situação de saúde da população local bem como para elaboração de estratégias que mantiveram as ações de vigilância epidemiológica do município.

Entre os principais resultados alcançados ao longo de dois anos de implantação do observatório estão a melhoria do conhecimento dos profissionais de saúde da Atenção Básica acerca do diagnóstico e manejo da sífilis, com adesão ao treinamento nas Unidades Básicas de Saúde de 92,6\%.

A fragmentação da RAS ainda não foi totalmente superada, mas os instrumentos implantados para melhoria da comunicação entre os serviços e o acompanhamento dos $\mathrm{RN}$ instaurado, reforçaram a integração do cuidado na sua produção ${ }^{7-8}$, acarretando no seguimento responsabilizado dos casos, o que evitou óbitos infantis em 2014, 2015 e 2016.

Nesse sentido, o observatório em Londrina acabou exercendo a tarefa proposta pelo Ministério da Saúde em 2015, que construiu diretrizes para constituição de "Comitês de Investigação de Transmissão Vertical", com objetivo de analisar eventos relacionados a agravos evitáveis e apontar medidas de intervenção para a sua redução em cada local, de modo a melhorar a qualidade da informação e permitir uma avaliação mais próxima da realidade da assistência prestada à gestante no pré-natal, parto e puerpério ${ }^{10-11}$.

\section{CONCLUSÃO}

A experiência apresentada descreve a medida de intervenção Observatório que como instrumento de gestão fortaleceu a comunicação entre os pontos da RAS e aperfeiçoou a qualidade do cuidado prestado ao binômio no pré-natal, parto e puerpério. Acredita-se que esse relato possa servir de modelo para ser replicado por outros serviços de saúde no Brasil para prevenir, diagnosticar e tratar não somente a sífilis, mas qualquer outro agravo, adaptando-se às realidades regionais dos serviços de saúde. =

\section{REFERÊNCIAS}

1. World Health Organization. Investment case for eliminating mother-to-child transmission of syphilis: promoting better maternal and child health and stronger health systems [Internet]. Geneva, 2012 [cited 2018 Feb 10]. Available from: http://apps.who. int/iris/bitstream/10665/75480/1/9789241504348_eng.pdf .

2. Organización Panamericana de la Salud. Iniciativa regional para la eliminación de la transmisión maternoinfantil de VIH y de la sífilis congénita en América Latina y el Caribe: documento conceptual [Internet]. Montevideo: CLAP/SMR; 2009 Sep [cited 2018 Mar 8] Disponible en: http://www.unicef.org/lac/overview_17863.htm.

3. Terry RF, Salm Junior JF, Nannei C, Dye C. Creating a global observatory for health R\&D. Science (New York, N.Y.) [Internet]. 2014 [cited 2018 Mar 8];345(6202):1302-1304. Available from: http:// science.sciencemag.org/content/345/6202/1302. doi: 10.1126/ science. 1258737

4. United Nations. Department of Economic and Social Affairs of the United Nations Secretariat. The Millennium Development Goals Report [Internet]. New York, 2015. Available from: http:// www.portalodm.com.br/publicacao/606/relatorio-dos-objetivos-de-desenvolvimento-do-milenio-2015

5. Brasil. Ministério da Saúde. Secretaria de Atenção à Saúde. Secretaria de Vigilância em Saúde. Portaria N 3.242, de 30 de dezembro de 2011. Dispõe sobre o Fluxograma Laboratorial da Sífilis e a utilização de testes rápidos para triagem da sífilis em situações especiais e apresenta outras recomendações. Diário Oficial da União, Brasília, 2011. Seção 1:51.

6. Prefeitura do Município de Londrina. Secretaria Municipal de Saúde. Conselho Municipal de Saúde. Plano Municipal de Saúde 2018-2021. Londrina, 2018. Disponivel em: https://www1. Iondrina.pr.gov.br/dados/images/stories/Storage/sec_saude/ Plano\%20Municial/plano_municipal_2018_2021.pdf
7.Cecilio, LCO. Escolhas para inovarmos na produção do cuidado, das práticas e do conhecimento: como não fazermos "mais do mesmo". Saúde e soc. [Internet]. 2012 Jun [S.I.] [citado 2016 Mar. 8]; 21(2):280-289. Disponivel em: http://www.revistas. usp.br/sausoc/article/view/48707/52780.doi:10.1590/S010412902012000200003.

8. Díaz J. Norma Conjunta de Prevención de la Transmisión Vertical del VIH y la sífilis: Programa Nacional de Prevención y Control de la infección por VIH/SIDA e ITS División de Prevención y Control de Enfermedades Subsecretaría de Salud Pública Ministerio de Salud. Rev chil infectol [Internet]. 2013 Jun [citado 2016 Mar. 8]; 30(3):259-302. Disponible en: http://www.scielo.cl/scielo. php?script=sci_arttext\&pid=S0716-10182013000300004\&Ing=en. Doi: 10.4067/S0716-1018201300030000

9. Paho. Pan American Health Organization. Elimination of Mother-to-Child Transmission of HIV and Syphilis in the Americas [Internet]. Washington, DC; 2015 [cited 2016 Mar 8]. Available from: http://iris.paho.org/xmlui/bitstream/handle/123456789/18372/9789275118702_eng.pdf?sequence=3\&isAllowed=y

10. Ministério da Saúde (BR). Secretaria de Vigilância em Saúde. Programa Nacional de DST e Aids. Transmissão Vertical do HIV e Sífilis: Estratégias para Redução e Eliminação [Internet]. Brasília, DF, 2014 [citado 2016 Mar 8]. Disponivel em: http://www.aids. gov.br/sites/default/files/anexos/publicacao/2014/56610/folder_transmissao_vertical_hiv_sifilis_web_pd_60085.pdf

11. Who.World Health Organization. Estrategia Mundial Del Sector De La Salud Contra Las Infecciones De Transmisión Sexual 2016-2021.[Internet]. Geneva, 2016 [citado 2019 Feb 20]. Disponible en: https://apps.who.int/iris/bitstream/handle/10665/250253/WHO-RHR-16.09-spa.pdf;jsessionid=7A1FF254ABD0A4A0032468A809B2AF48? sequence $=1$ 